\title{
Simulación DEL TIEMPO DE CALENTAMIENTO DEL FUELOIL PARA DETERMINAR VISCOSIDAD ÓPTIMA DE BOMBEO
}

\author{
Fuel Oil heating time simulation to \\ DETERMINE OPTIMAL PUMPING VISCOSITY
}

\author{
Sandra Elvira Fajardo-Muñoz ${ }^{1}$, Galo Jaime Páez-Fajardo,*
}

\begin{abstract}
Resumen
Con el fin de estimar el tiempo requerido para alcanzar la viscosidad óptima de bombeo de un material altamente viscoso, se lleva a cabo una simulación del calentamiento de Heavy Fuel Oil (HFO) ecuatoriano mediante serpentines en tanques a bordo. Se estudia la transmisión y transferencia de calor a través de un fluido altamente viscoso como el HFO, se incluyen procesos de conservación energética, conservación de masa y conservación de momento lineal sobre fluidos viscosos. Adicionalmente se incluyen procesos de difusión y convección energética. Finalmente, se determina que la evolución temporal de la temperatura promedio no muestra dependencia al perfil de calentamiento del vapor de agua en serpentines, pero sí una dependencia lineal en el tiempo.
\end{abstract}

Palabras clave: viscosidad de crudo, fluido viscoso, sistema calentamiento, serpentines, crudo pesado ecuatoriano.

\begin{abstract}
In order to estimate the time required to reach the average temperature of a highly viscous material with which optimal pumping viscosity is obtained, a simulation of an on-board coil heating system on Ecuadorian Heavy Fuel Oil (HFO) was conducted. Heat transmission and heat transfer is studied through a highly viscous fluid such as HFO; processes such as energy conservation, mass conservation, and conservation of momentum on viscous fluids are included. Additionally, processes of energy diffusion and energy convection must be included. It was found that temporal evolution of mean temperature does not have dependence with the steam heating profile in coils, but indeed it has a linear dependence in time.
\end{abstract}

Keywords: Ecuadorian Heavy Fuel Oil, Crude viscosity, Viscous fluid, Heating system, Serpentines.

\footnotetext{
${ }^{1}$ Facultad de Ingeniería Química, Universidad de Guayaquil, Guayaquil - Ecuador.

${ }^{2, *}$ Vicerrectorado Académico, Universidad Regional Amazónica IKIAM, Napo - Ecuador. Autor para correspondencia : galo.paez@ikiam.edu.ec
} 


\section{Introducción}

Heavy Fuel Oil (HFO) o búnker, como se conoce en Ecuador, es un combustible residual de alto poder calorífico [1] y de bajo costo relativo. Por lo general, y según las especificaciones internacionales, el HFO ecuatoriano se cataloga como fueloil N. ${ }^{\circ} 6$. Sus aplicaciones son numerosas y se presentan en diferentes tipos de industrias. Esencialmente, el HFO se usa en motores de navíos mercantes, en combustión por vaporización en calderos industriales [2], así como en centrales termoeléctricas, entre otros.

La composición de este crudo varía sustancialmente debido al método de producción del HFO y a la calidad del petróleo del proceso de refinación [1]. Como consecuencia, sus propiedades termodinámicas tales como la viscosidad, la densidad y la capacidad calórica también muestran una común variabilidad [3] y dependencia al proceso de producción. Esta característica del crudo induce incertidumbre en la determinación de funciones características en el modelamiento computacional del HFO, sin embargo, la inexactitud computacional puede ser mitigada con un amplio proceso de caracterización del búnker ecuatoriano.

Para el caso ecuatoriano, el HFO es el resultado de diluir combustibles más ligeros como el diésel con el producto que se obtiene al llevar a cabo el proceso de cracking térmico en el residuo de la torre de fraccionamiento a presión atmosférica. Esta etapa garantiza obtener las exigencias técnicas y de bombeo dinámicas del comprador. Como consecuencia natural de este proceso de refinamiento, en general, el HFO es catalogado como un fluido altamente viscoso $[4,5]$; por lo que para transportarlo fácilmente desde tierra al buque o viceversa es necesario reducir su viscosidad drásticamente, para esto se requiere introducir un proceso de precalentamiento y calentamiento tal como se propone y simula computacionalmente en $[2,3,6]$.

Uno de los procesos de calentamiento a bordo más utilizados en la industria es el calentamiento mediante vapor de agua $[7,8]$. El proceso se basa principalmente en la transferencia de calor desde el vapor de agua hacia el HFO mediante el contacto de este último con serpentines de acero inoxidable distribuidos uniformemente en la base del contenedor del combustible [7]. Este sistema utiliza vapor de agua a temperaturas que bordean los 150 grados centígrados [6], por ende, requiere un alto consumo de combustible para lograr la temperatura que permite alcanzar la viscosidad óptima de bombeo del HFO, lo que se traduce en un alto costo de calentamiento.

En este contexto, el principal problema de la industria ecuatoriana de transporte de $\mathrm{HFO}$ radica en que se desconoce el tiempo con el cual el combustible alcanza su punto de viscosidad óptima de bombeo.
En este trabajo, un análisis computacional del flujo dinámico de calor sobre materiales viscosos $[2,3,6]$ es utilizado para sobrellevar este inconveniente técnico. Particularmente, se considera al HFO como fluido compresible, se introduce procesos de transporte energético por convección y difusión. Adicionalmente, este trabajo a diferencia de otros en simulación como en [3] o en [6] incluye un micromovimiento ascendente de las capas cercanas a la fuente de calentamiento producto de la variación de densidad, así como considerar en el modelado perfiles de calentamiento del vapor de agua tanto lineales (flujo calórico sin restricción) como exponenciales (restricción sistemática del calentador). Finalmente, se analiza su influencia sobre la capacidad de calentamiento de los serpentines.

\section{Materiales, fuentes y métodos}

El fenómeno que se analiza al estudiar el calentamiento de HFO es la distribución energética sobre material como consecuencia de introducir tres procesos fundamentales de transporte: la conservación de energía, la conservación de momento y la conservación de masa. Para todos los procesos considerados, el volumen de control es aquel que ocupa el combustible dentro del contenedor de almacenamiento. El volumen diferencial de referencia forma parte del fluido viscoso y se expresa en coordenadas rectangulares diferenciales. Adicionalmente, se considera que el contenedor que almacena el combustible es rectangular prismático y de dimensiones definidas.

La conservación de masa no es más que establecer cuánta masa existe dentro del volumen diferencial de referencia. Es decir, el cambio temporal de la cantidad de masa que existe dentro del volumen es consecuencia de la cantidad de masa que entra y que sale de dicho volumen al transcurrir el tiempo [9]. Esta idea se expresa en forma diferencial mediante la siguiente expresión:

$$
\frac{\partial \rho(T)}{\partial t}+\nabla \cdot(\rho \vec{v})=0
$$

Donde $\rho$, es la densidad del fluido, y $\vec{v}$ es la velocidad del fluido. El primer término se asocia al cambio temporal de la materia dentro del volumen de control, mientras que el segundo término se relaciona con el flujo neto de masa a través del volumen de control.

$\mathrm{Al}$ igual que en la conservación de la masa, la energía en un fluido se conserva cuando el cambio temporal de la energía interna dentro del volumen diferencial de referencia es consecuencia del flujo neto de calor por conducción y por convección sobre dicho volumen. Adicional a esta condición, se introduce como única pérdida de energía del sistema, al rozamiento viscoso en la vecindad de dicho volumen [10]. Este comportamiento 
del flujo de energía se expresa en forma diferencial mediante la siguiente expresión:

$$
\rho(T) c_{p} \frac{\partial T}{\partial t}+\nabla \cdot \vec{q}-\mu(T) \phi=0
$$

Donde $\vec{q}$ definido como $\vec{q}=-k(T) \nabla T+\rho H \vec{v}$ es el vector de flujo de calor conductivo y convectivo respectivamente, $\mathrm{c}_{\mathrm{p}}$ el coeficiente de calor específico del HFO, $\mathrm{T}$ el campo escalar de temperatura que depende del tiempo y de la posición, $\mu$ es el parámetro de viscosidad del combustible que mide la facilidad con que un fluido se desplaza con respecto a su vecindad, $\phi$ es la función de disipación viscosa que representa el rozamiento en las capas laterales del fluido al desplazarse $[10,11]$.

La variación temporal de la cantidad de movimiento total del sistema en el volumen referencial, es consecuencia de la acción de tres fuerzas generales sobre el fluido, la presión que actúa sobre las paredes de región diferencial de análisis, el efecto del rozamiento en las inmediaciones del volumen y la acción de la fuerza de gravedad [11]. Estos efectos se resumen en la siguiente expresión:

$$
\rho(T) \frac{d \vec{v}}{d t}=-\nabla P+\nabla \cdot \tau+\rho(T) \vec{g}
$$

Donde $\vec{v}$ es la velocidad del fluido, $\mathrm{P}$ es la presión, $\tau$ es el tensor de deformación en las inmediaciones del volumen de referencia, y $\vec{g}$ la aceleración de la gravedad. Para todos los casos, el operador $\nabla$ es el operador vectorial gradiente tridimensional permitiendo que el operador de cambio temporal $\frac{d}{d t}$ se define como $\frac{d}{d t} \equiv \frac{\partial}{\partial t}+\vec{v} \cdot \nabla$.

\subsection{Adaptaciones y consideraciones sobre el sistema}

El HFO es un combustible pesado con muchas de sus propiedades termodinámicas como las antes mencionadas que dependen esencialmente de su temperatura [1-4], es por esto que es más factible representar, para este caso, esa dependencia de temperatura en su viscosidad, su densidad y su capacidad calórica. Por lo tanto, dichas funciones termodinámicas variarán únicamente como consecuencia de una variación en T. Es decir, la densidad $\rho$ en $\left[\mathrm{kg} / \mathrm{m}^{3}\right]$ variará según la expresión propuesta en [6]:

$$
\rho(T)=0,16 c_{1} c_{2}-\left(1+\left[1-\frac{T}{c_{3}}\right]^{c_{4}}\right)
$$

Donde $\mathrm{c}_{1}=0,537, \mathrm{c}_{2}=0,261, \mathrm{c}_{3}=568,7, \mathrm{y} \mathrm{c}_{4}=0,280$ son coeficientes de ajustes y $\mathrm{T}$ la temperatura en grados Kelvin. El factor 0,16 corresponde a un factor que permite estimar la densidad del fueloil ecuatoriano con la ecuación (4).

La capacidad calórica $c_{p}$ se obtiene al derivar con respecto a la temperatura la ecuación de entalpía de [6], así obtenemos:

$$
\begin{array}{r}
c_{p}(T)=6,34 R\left(A_{1}+A_{2} T+A_{3} T^{2}\right. \\
\left.+A_{4} T^{3}+A_{5} T^{4}\right)
\end{array}
$$

Donde $\mathrm{A}_{1}=1,25 \cdot 10^{1}, \mathrm{~A}_{2}=-1,01 \cdot 10^{-2}$, $\mathrm{A}_{3}=2,22 \cdot 10^{-4}, \mathrm{~A}_{4}=-2,85 \cdot 10^{-7}, \mathrm{~A}_{5}=1,12 \cdot 10^{-10}$ son coeficientes de ajuste propuestos en [12], mientras que $\mathrm{R}=8,3144[\mathrm{~J} / \mathrm{molK}]$ es la constante de los gases, 6,34 es la constante de ajuste que permite a la ecuación (5) modelar la capacidad calórica del fueloil ecuatoriano. Así como en el caso anterior, $\mathrm{T}$ es la temperatura en grados Kelvin.

El cambio de la viscosidad con respecto a la temperatura del búnker ecuatoriano es uno de las especificaciones normalmente exigidas por compradores nacionales e internacionales. Previo a una cordial petición, fácilmente se puede obtener datos experimentales del perfil de viscosidad versus temperatura del fueloil ecuatoriano, esto permitió generar un ajuste de los mismos a una ecuación de la forma:

$$
\mu(T)=D T^{A} e^{\left[\frac{B}{T}+\frac{C}{T^{2}}\right]}
$$

Con $\mu[\mathrm{kg} / \mathrm{ms}]$ como la viscosidad dinámica del búnker y coeficientes de ajuste $\mathrm{A}=-5,79, \mathrm{~B}=8496,41$, $\mathrm{C}=110,48, \mathrm{y} \mathrm{D}=522,64$. Como en los otros casos, $\mathrm{T}$ es la temperatura expresada en grados Kelvin. La bondad de ajuste del proceso es $\mathrm{X}^{2} / \mathrm{n}=0,0143$ el cual se realizó con 4 grados de libertad.

A pesar de que el modelo computacional considera al contenedor del HFO con forma de prisma rectangular, de las tres dimensiones del sistema de ecuaciones, la única que muestra representación relevante del fenómeno es la manera de distribución de la energía calórica desde los serpentines, ubicados en el fondo del tanque, hacia la capa superficial del combustible, ubicado en las cercanías del tope del tanque; es decir, la variación de temperatura en el tiempo a lo alto del tanque (coordenada $\mathrm{z}$ ).

En otras palabras, la capa asociada a cierto nivel de altura muestra una distribución uniforme energética solo alterada por flujos perpendiculares de energía a dicha capa o como también es conocido, el sistema muestra simetría rectangular superficial. Más detalladamente, al someterse el HFO al proceso de calentamiento, se evidencia cambios significativos exclusivamente cuando se analiza el transporte de energía a diferentes alturas z con respecto al fondo del contenedor. Por lo tanto, el sistema solo considerará a la altura como única dimensión de análisis.

Como consecuencia, en las ecuaciones (1), (2) y (3), el operador gradiente se reescribirá en la forma $\nabla=\frac{\partial}{\partial z}$, el campo escalar de temperatura $\mathrm{T}$ dependerá netamente del tiempo y la posición de altura z, a su vez, la velocidad así como la función de disipación mostrarán solo componentes en la dirección z, quedan 
reescritos tanto como $\vec{v}=w \vec{k}$ y como en ecuación (7) respectivamente [10].

$$
\phi=2\left(\frac{\partial w}{\partial z}\right)^{2}
$$

Adicionalmente, el tensor de deformación $\tau$, se reescribirá en forma propuesta en la ecuación (8), el cual eventualmente muestra dependencia con el coeficiente de expansión volumétrica $\beta$.

$$
\nabla \cdot \tau=\frac{4}{3} \mu(T) \frac{\partial^{2} w}{\partial z^{2}}+\frac{4}{3} \beta w\left(\frac{\partial T}{\partial z}\right)^{2} \frac{\partial \mu}{\partial T}
$$

En el espacio encerrado por el volumen de control, no existe una fuente de masa de HFO, es decir, no existe proceso que adicione masa deliberadamente dentro del tanque. Esta consideración permite excluir al factor $\frac{\partial \rho(T)}{\partial t}$ de la ecuación 1 .

La primera capa del material, ubicada en el fondo del reservorio, captura en su totalidad la energía proveniente de los serpentines de calentamiento por difusión energética netamente; como consecuencia, esta capa experimenta un cambio de densidad que genera una fuerza ascendente transmitiendo cinética a la capa subsiguiente. La segunda capa experimentará el mismo efecto transmitiendo movimiento a la tercera capa y así sucesivamente. El efecto diferencial de dicha fuerza es producto de la diferencia de densidad por cambio térmico, es decir, se tiene una especie de fuerza boyante térmica, que se expresa mediante la siguiente expresión.

$$
-\nabla P+\rho \vec{g}=-\rho \beta \Delta T \vec{g}
$$

Que incluyendo las consideraciones de unidimensionalidad, la ecuación (9) se reescribe en:

$$
-\frac{\partial P}{\partial z}-\rho g=\rho \beta g \frac{\partial T}{\partial z} \Delta z
$$

En este trabajo, a diferencia de otros, se considera que este efecto permite una dinámica ascendente más rápida de la energía, por ende, el término no es descartado de la simulación.

En conclusión, el sistema en su totalidad llega a particularizarse con las condiciones exigidas por el proceso de calentamiento transformando la ecuación (1) a:

$$
w \beta \frac{\partial T}{\partial z}=\frac{\partial w}{\partial z}
$$

La ecuación (2) a:

$$
\rho c_{p}\left(\frac{\partial T}{\partial t}+w \frac{\partial T}{\partial z}\right)=k \frac{\partial^{2} T}{\partial z^{2}}+2 \mu\left(\frac{\partial w}{\partial z}\right)^{2}
$$

y la ecuación (3) a:

$$
\begin{array}{r}
\rho\left(\frac{\partial w}{\partial t}+w \frac{\partial w}{\partial z}\right)=\rho \beta g \Delta T+\frac{4}{3} \mu(T) \frac{\partial^{2} w}{\partial z^{2}} \\
+\frac{4}{3} \beta w\left(\frac{\partial T}{\partial z}\right)^{2} \frac{\partial \mu}{\partial T}
\end{array}
$$

\subsection{Condiciones de frontera}

La primera capa del HFO se encuentra en constante contacto con los serpentines de acero inoxidable; es decir, la potencia energética $p$ que esta percibe proviene del calor del vapor de agua con potencia $\dot{E}$, en unidades internacionales, luego de atravesar el acero de los serpentines. Por tanto, la condición de frontera cuando $\mathrm{z}=0$ se expresa en la ecuación (14):

$$
m_{a} c_{p} \frac{\partial T(0, t)}{\partial t}=p
$$

Donde $m_{a}=\rho A \nabla h$ es la masa de la capa superficial del fondo, $A$ el área inferior del tanque contenedor y $\nabla h$ la finesa de la primera cada que es igual al ancho de la grilla espacial en la simulación. La temperatura a lo largo de los serpentines se distribuye desde el radio interior de los serpentines, donde hace contacto con el vapor de agua, hacia el radio exterior, donde hace contacto con el búnker, esta distribución sigue la expresión:

$$
\frac{1}{r} \frac{\partial}{\partial r}\left(r k_{\text {acero }} \frac{\partial T}{\partial r}\right)+\dot{e}=0
$$

Donde $\dot{e}=\frac{\dot{E}}{\pi r_{\text {out }}^{2} L}$ es la densidad de potencia aportada por el vapor de agua para el régimen lineal y $\dot{e}=\frac{\dot{E}}{\pi r^{2} L}$ es la densidad de potencia aportado por el vapor de agua para el régimen exponencial, $r_{i n}$ es el radio interno de los serpentines y $r_{\text {out }}$ el radio externo de los serpentines. De esta relación diferencial, el valor de p se encuentra según el régimen de calentamiento que se considere. Para cualquiera de ambos casos, cuando $r=r_{\text {in }}$ entonces $k_{\text {acero }} \frac{\partial T}{\partial r}=\frac{\dot{E}}{2 \pi r_{i n} L}$ y cuando $r=r_{\text {out }}$ entonces $k_{\text {acero }} \frac{\partial T}{\partial r}=\frac{p}{2 \pi r_{\text {out }} L}$. De esta manera, para el régimen exponencial $p=\dot{E}\left(1-\ln \left(\frac{r_{\text {out }}}{r_{\text {in }}}\right)^{2}\right)$ y para el régimen lineal $p=\dot{E}\left(\frac{r_{i n}}{r_{\text {out }}}\right)^{2}$. En todo caso, la potencia de energía que brinda el vapor de agua $\dot{E}$ se puede escribir según la ecuación (16), esto es, mediante su poder calorífico $f$ como se lo propone en [8], $2250 \cdot 10^{3}<f<3120 \cdot 10^{3}[\mathrm{~J} / \mathrm{kg}]$, la velocidad con que el vapor viaja dentro de la tubería $v=15,4 \mathrm{~m} / \mathrm{s}$ [6], el área transversal de la tubería $A$ y la densidad del vapor de agua $\rho_{V}$.

$$
\dot{E}=f v A \rho_{v}
$$




\section{Resultados y discusión}

Se predecía que al considerar dos formas de distribución energética distintas a lo largo del grosor de los serpentines (régimen lineal y régimen exponencial), se produzca una diferencia relevante en el promedio de la temperatura distribuida a lo alto del HFO conforme aumenta el tiempo de calentamiento. Sin embargo, a distintos niveles de ocupación del tanque, para ambos regímenes, la dependencia del promedio de la temperatura con respecto al aumento del tiempo de calentamiento muestra un comportamiento similar, lineal y creciente. Este comportamiento se observa en las Figuras 1 y 2.

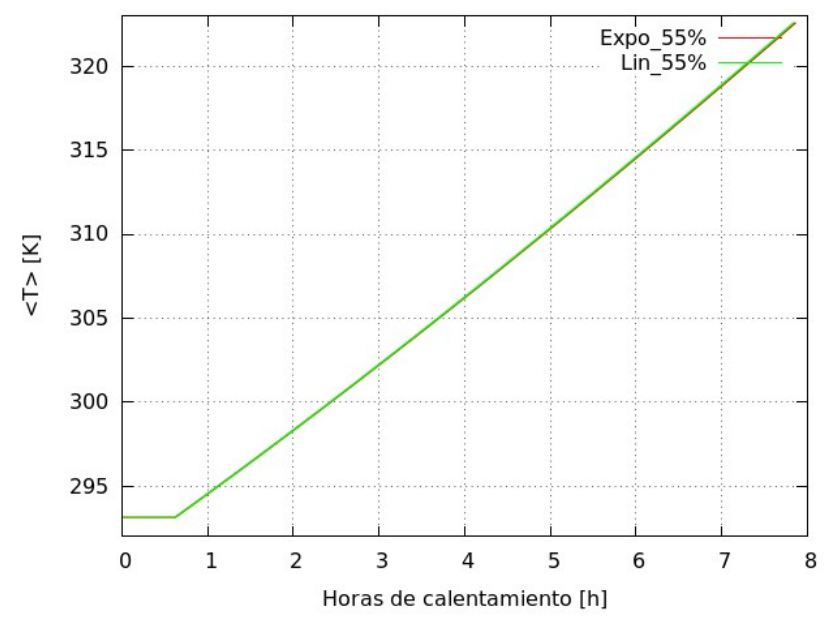

Figura 1. Cambio del promedio de la temperatura durante el calentamiento, capacidad de ocupación al $55 \%$

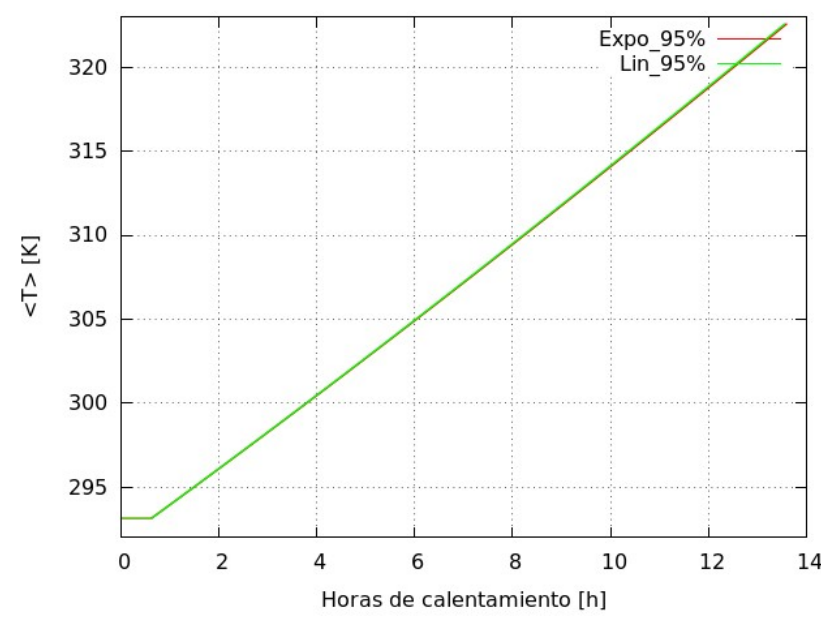

Figura 2. Cambio del promedio de la temperatura durante el calentamiento, capacidad de ocupación al $95 \%$

Trabajando con la ecuación (15), es evidente que la potencia con que se calienta la capa inicial inferior del combustible es diferente para los distintos regímenes.
Matemáticamente se observa que la potencia exponencial es mayor a la lineal. Por ende, la distribución de la temperatura a lo alto del tanque y conforme el tiempo transcurrido será de mayor magnitud para el proceso exponencial en regiones cercanas al fondo del tanque complementado a su vez con velocidades de ascenso del flujo energético también mayor en sectores alejadas del fondo. Sin embargo, el proceso de promediar las temperaturas de distintas alturas solapó las diferencias espaciales en la distribución térmica (Figura 3), provocando que para ambos casos el cambio de la temperatura promedio sea apenas evidente, (Figuras 1 y 2).

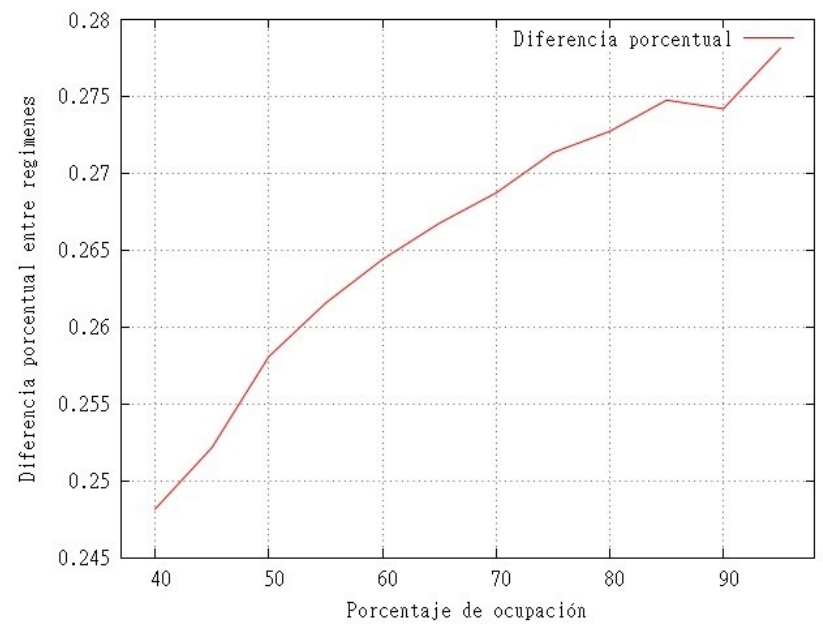

Figura 3. Diferencia porcentual del tiempo de calentamiento del proceso exponencial con respecto al proceso lineal

La diferencia máxima entre los dos perfiles de calentamiento es menor al 0,29\%, mientras que la mínima oscila por $0,25 \%$. En consecuencia, diferenciar el perfil de calentamiento no indujo a un cambio relevante en el proceso de calentamiento promediado ya que en los resultados de los métodos se diferencian en promedio en apenas un 0,26\%.

La simulación del calentamiento del hidrocarburo calcula, mediante iteraciones en el tiempo, la distribución de la temperatura a lo alto del fueloil. Para cada iteración temporal se calcula la temperatura promedio en el tanque para posteriormente determinar la viscosidad del material para esa temperatura. El programa se detendrá una vez que la viscosidad de material se acerque a la viscosidad objetivo de $454,5 \mathrm{cS}$ a una temperatura de $322,57 \mathrm{~K}\left(120,96{ }^{\circ} \mathrm{F}\right)$ para el caso ecuatoriano.

Como es de esperarse a medida que aumenta la cantidad de material a ser calentado, el tiempo de calentamiento para alcanzar la viscosidad objetivo también aumenta. Este comportamiento es confirmado por la simulación y se evidencia en la Figura 4. Por otro lado, 
se esperaba que la temperatura promedio para el régimen exponencial crezca a una tasa superior a la que observaríamos para el caso lineal. Los resultados de las simulaciones verifican débilmente lo esperado, puesto que, para el caso lineal, el tiempo de calentamiento óptimo crece a una tasa de 0,1424 horas por cada porcentaje de ocupación del tanque de fueloil, mientras que parte el régimen exponencial se calcula una tasa de aumento del tiempo óptimo de calentamiento de 0,1571 horas por cada unidad de porcentaje de ocupación del tanque.

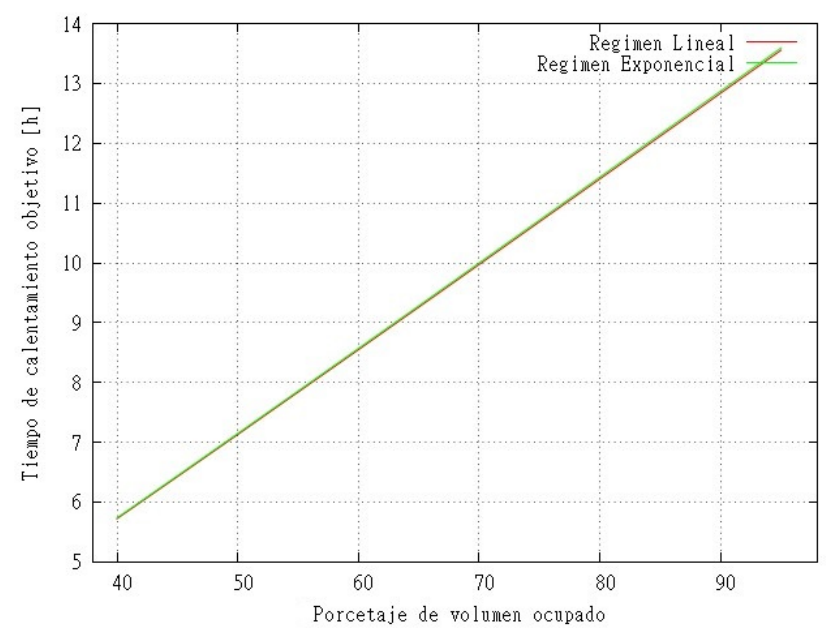

Figura 4. Resultado del sistema de simulación, tiempo de calentamiento para distintos niveles de ocupación

Adicionalmente se esperaba observar una función de la temperatura promedio en dependencia del tiempo de calentamiento que varíe acorde al régimen del perfil de temperatura del vapor de agua, pero se obtuvo lo contrario. Como se observa en la Figura 4, para ambas consideraciones de calentamiento, cuando el porcentaje de llenado bordea el $40 \%$, el tiempo de calentamiento oscila entre 5 a 6 horas mientras que cuando el tanque se llena en un $95 \%$, el tiempo de calentamiento oscila entre 13 a 14 horas. Este comportamiento se promedió y generalizó en la ecuación (17) con un parámetro de aproximación de $X^{2} / n=7,7 \cdot 10^{-5}$ y usando 10 grados de libertad.

$$
t(P)=0,1427 P+0,002
$$

Para este caso t es el tiempo de calentamiento descrito en horas y $P \%$ es el porcentaje del tanque ocupado por el combustible. Las constantes son válidas únicamente para un tanque con dimensiones específicas de 11,47 metros de largo, 30,0 metros de ancho y 8,3 metros de alto. Es recomendable que solo se trabaje con rango de porcentaje de llenado de entre $38 \%$ y el $96 \%$.

La poca diferencia que se observa para las temperaturas promedios en la Figura 4 se explica cuando se realiza un análisis de valor inicial para la ecuación diferencial parcial de la distribución de la temperatura a distintos niveles de altura. La forma diferencial de distribuir la temperatura es igual para ambos casos, (ecuaciones (11), (12) y (13)), mas sin embargo, la diferencia radica netamente por incluir valores iniciales distintos correspondientes a los regímenes del perfil térmico del vapor de agua.

Ya se conoce que el régimen exponencial permite una mayor potencia de calentamiento que se deriva tanto en una capa superficial inicial del combustible que se caliente con mayor rapidez como en una velocidad de ascenso energético con mayor magnitud por convección que se traduce simultáneamente en una mayor transmisión de energía hacia las capas alejadas de la fuente y en una tasa de disipación por fricción mayor. Por otro lado, el régimen lineal provee de menor potencia que el exponencial, por ende, bajo este régimen, la capa inicial se calentará con menor rapidez y la velocidad de ascenso por convección mostrará una menor magnitud, sin embargo, esto permitirá perder menor calor por disipación por fricción.

Evidentemente, estas diferencias en la dinámica de la distribución de energía se atenúan para bajos porcentajes de ocupación porque en estos casos las capas superficiales del combustible están cerca de la fuente de calor despreciando el efecto de distribución por velocidad convectiva y favoreciendo al efecto por difusión, mientras que a altos porcentajes de ocupación el efecto por convección empieza a ser relevante en la distribución de energía de tal manera que en promedio se observa una mayor temperatura para el caso exponencial que para el caso lineal.

\section{Conclusiones y recomendaciones}

Aun cuando en la literatura se puede encontrar una amplia gama de aplicación de la dinámica de fluido computacional $[2,3,6,11]$, (CFD) por sus siglas en inglés. Debido a la difícil tarea de establecer una generalización de las propiedades termodinámicas para todos los combustibles HFO, los programas de simulación mediante CFD están forzados a particularizar su aplicación hacia ciertos tipos de combustibles con propiedades innatas de dicho crudo, por ende, sus conclusiones solo son aplicables para aquellos HFO que posean el mismo proceso de refinación y la misma calidad de petróleo involucrado en la refinación. Por ejemplo, en [6] se estudia el diseño de tanques de calentamiento y longitud de serpentines para HFO griego con propiedades termodinámicas que difieren enormemente con las del búnker ecuatoriano debido a que el crudo de refinación griego es más ligero que el local, por ende, estos tanques no trabajarán con la misma eficiencia que con el búnker ecuatoriano. Es por este motivo, que urge la necesidad de empezar a generar información termodinámica del crudo local para que 
los procesos de simulación, diseño y construcción de tanques y de procesos de calentamiento reflejen con mayor exactitud la realidad del HFO local.

Sin embargo, los procesos de simulación pueden compartir ciertas propiedades aun cuando son particularizados para HFO distintos. Es por este motivo que ciertas consideraciones computacionales relacionadas a la matemática diferencial del problema propuestas en [2] y [11] fueron acogidas, modificadas y acoplados en este trabajo. Por este motivo, esta simulación reproduce en cierta medida la dinámica térmica y energética de medios viscosos propuesta en [11] aun cuando la simulación se llevó a cabo sobre el crudo pesado ecuatoriano.

En primera instancia, el tiempo de calentamiento óptimo es similar sin importar el perfil con el que se calienta el vapor de agua del sistema de calentamiento, diferencias de apenas un 0,26\% garantizan esta similitud. Adicionalmente, la velocidad con que se transmite la energía es distinta para los diferentes porcentajes de ocupación del tanque, producto de introducir diferentes regímenes de calentamiento (lineal o exponencial). A mayor porcentaje los procesos convectivos retoman relevancia mientras que a menor porcentaje de ocupación basta con considerar únicamente procesos difusivos de energía. Finalmente, bajo los parámetros y supuestos establecidos en este estudio, se concluye que existe una relación lineal expresada por la ecuación (17) entre el tiempo promedio de calentamiento del HFO y la cantidad de material que llena los tanques.

Por otra parte, consideramos necesario generar más estudios que permitan que las características del material ecuatoriano puedan ser altamente representadas por las ecuaciones y constantes de ajuste establecidas para las ecuaciones (4), (5), y (6); por consiguiente, al momento, la ecuación (17) solo nos garantiza una primera aproximación del estudio del fenómeno de calentamiento de HFO particular, mas no determinante o generalizable. En consecuencia, es recomendable incluir más estudios de caracterización del búnker ecuatoriano para permitir generar mejores aproximaciones computacionales y por ende relaciones del tipo de ecuación (17) más exactas, confiables y generalizables.

En ciertos trabajos obvian el uso de la ecuación de conservación de momento por considerar irrelevante el flujo másico ascendente, aun así, en este estudio sí se consideró esta ecuación y hemos observado que los resultados se aproximan a los empíricamente esperados. Es recomendable realizar un estudio experimental para corroborar parcial o totalmente los resultados estipulados por este proceso de simulación, además, es aconsejable establecer un proceso de generalización del sistema de simulación y adaptar su parametrización a las propiedades térmicas del crudo ecuatoriano para los diferentes procesos de producción del mismo. Obteniendo una generalización de esta sim- ulación que incluya las diferentes características del crudo ecuatoriano.

Existe una gran falta de información de caracterización del HFO o crudo pesado ecuatoriano en propiedades no consideradas por el ámbito comercial. Es altamente recomendable centrar estudios en la caracterización de las propiedades termodinámicas como la densidad, la capacidad calórica, la viscosidad dinámica, la viscosidad cinética, la entalpía del material y sus dependencias respecto al proceso de producción del HFO ecuatoriano. Esta proyección para el futuro abarcará un rango de temperatura bastante amplio que permitirá trabajar eventualmente en simulaciones más precisas y ajustadas al medio local.

\section{Referencias}

[1] J. S. Arroyo, "Aprovechamiento del recurso biomasa a partir de los desechos de madera para una caldera de vapor," in Proyecto de Titulación. Universidad Politécnica Salesiana, Quito - Ecuador, 2015.

[2] C. Chryssakis, K. Pantazis, and L. Kaiktsis, "Combustion modeling with heavy fuel oil for large marine diesel engine applications," in Conseil international des machines a combustion, Bergen, 2010.

[3] N. Kyriakides, C. Chryssakis, and L. Kaiktsis, "Development of a computational model for heavy fuel oil for marine diesel engine applications," in 19th Int. Multidimensional Engine Modeling User's Group Meeting at the SAE Congress, Detroit, 2009.

[4] O. Alomair, M. Jumaa, A. Alkoriem, and M. Hamed, "Heavy oil viscosity and density prediction at normal and elevated temperatures," $J$ Petrol Explor Prod Technol, vol. 6, no. 2, pp. 253$263,2015$.

[5] Q. Chen, M. Wang, N. Pan, and Z.-Y. Guo, "Optimization principle for variable viscosity fluid flow and its application to heavy oil flow drag reduction," Energy Fuels, vol. 23, no. 9, pp. 4470-4478, 2009.

[6] G. Gavriil, G. N. Prodromidis, J. Pitsilos, and F. A. Coutelieris, "Steaming heat coils for heating up marine heavy fuel oil," in 8th GRACM International Congress on Computational Mechanics, Volos, 2015.

[7] The Standard P\&i Club, "A master's guide to: using fuel oil onboard ship," Londres, 2012.

[8] R. M. Butler, G. S. M. Butler, and H. Y. Lo, "Theoretical studies on the gravity drainage of 
heavy oil during in-situ steam heating," in 29th Canadian Chemical Engineering Conference in Sarnia, Ontario, 1979.

[9] Y. A. Cengel and J. M. Cimbala, Mecánica de fluidos. Fundamentos y aplicaciones, Segunda ed., P. E. Roig, Ed. Mc Graw-Hill, México DF, 2012.

[10] Y. Cengel and A. Ghajar, Transferencia de calor y masa, Cuarta ed., P. E. Roig, Ed. Mc Graw-Hill, México D.F., 2012.

[11] J. D. Anderson, Jr, Computational Fluid Dynamics: An Introduction. Springer, 2009.

[12] B. McBride, S. Gordon, and M. Reno, "Coefficients for calculating thermodynamic and transport properties of individual species," 1993. 